\title{
microRNA-200a-3p enhances mitochondrial elongation by targeting mitochondrial fission factor
}

\author{
Heejin Lee ${ }^{1, \#}$, Hyosun Tak ${ }^{1, \#}$, So Jung Park ${ }^{2}$, Yoon Kyung Jo ${ }^{2}$, Dong Hyung Cho ${ }^{2, *}$ E Eun Kyung Lee ${ }^{1, *}$ \\ ${ }^{1}$ Department of Biochemistry, College of Medicine, The Catholic University of Korea, Seoul 06591, ${ }^{2}$ Department of Gerontology, Graduate \\ School of East-West Medical Science, Kyung Hee University, Yongin 17014, Korea
}

\begin{abstract}
Mitochondria play pivotal roles in the ATP production, apoptosis and generation of reactive oxygen species. Although dynamic regulation of mitochondria morphology is a critical step to maintain cellular homeostasis, the regulatory mechanisms are not yet fully elucidated. In this study, we identified miR-200a-3p as a novel regulator of mitochondrial dynamics by targeting mitochondrial fission factor (MFF). We demonstrated that the ectopic expression of miR-200a-3p enhanced mitochondrial elongation, mitochondrial ATP synthesis, mitochondrial membrane potential and oxygen consumption rate. These results indicate that miR-200a-3p positively regulates mitochondrial elongation by downregulating MFF expression. [BMB Reports 2017; 50(4): 214-219]
\end{abstract}

\section{INTRODUCTION}

Mitochondria play essential roles in balancing cellular energy homeostasis as well as regulation of apoptosis (1-3). Tight regulation of mitochondrial morphology in response to various cellular stimuli is critical to maintain mitochondrial function. Mitochondria continuously change their morphologies by dividing (fission) or elongating (fusion) each other. Several key proteins regulating mitochondrial morphology have been identified. Dynamin-related protein (DRP1), mitochondrial fission 1 protein (FIS1), and mitochondrial fission factor (MFF) promote mitochondrial fragmentation, while mitofusin $1 / 2$ (MFN1/2), and optic atrophy 1 (OPA1) lead to mitochondrial elongation (3-6). Relative expression levels or post-translational

*Corresponding authors. Eun Kyung Lee, Tel: +82-2-2258-7295; Fax: +82-2-596-4433; E-mail: leeek@catholic.ac.kr, Dong Hyung Cho, Tel: +82-31-201-2311; Fax: +82-31-201-2312; E-mail: dhcho @khu.ac.kr

${ }^{\#}$ These authors contributed equally to this work.

https://doi.org/10.5483/BMBRep.2017.50.4.006

Received 14 January 2017, Revised 16 January 2017, Accepted 31 January 2017

Keywords: microRNAs, miR-200a-3p, Mitochondria dynamics, Mitochondria fragmentation, Mitochondrial fission factor modifications of key regulatory proteins are responsible for dynamic changes in mitochondrial morphology (3, 4, 6-9). Although recent reports have shown that post-translational regulatory mechanisms to control the quality of key proteins including phosphorylation (10), de-acetylation (11), and ubiquitination (12), detailed mechanism governing mitochondrial morphology is not fully understood.

microRNAs (miRNAs), small non-coding RNAs (18-22 nt long) downregulate gene expression by destabilizing target mRNAs or inhibiting translation, thereby affecting various cellular processes such as cell proliferation, survival, death, and differentiation (13-24). miRNA expression could be regulated in time- and tissue-specific manners, and differential regulation of miRNAs is closely related to the pathogenesis of diseases $(14,19,25-28)$. Recent studies have shown that miRNAs regulate dynamic changes of in the mitochondria morphology by regulating the expression of several key proteins governing mitochondrial dynamics. For example, miR-483-5p and miR-484 are responsible for suppressing mitochondrial fission by targeting FIS1 (29, 30). miR-499 affects mitochondrial dynamics by down-regulating DRP1 expression (31). miR-140 and miR-19b have been reported to decrease mitochondrial elongation through targeting MFN1, and miR-106, miR-195, and miR-761 down-regulate MFN2 expression (32-36). miR-27, miR-761, and miR-593 are responsible for mitochondrial dynamics by downregulating MFF expression (37-39).

In this study, we investigated the role of miR-200a-3p as a novel factor governing mitochondrial dynamics by targeting MFF, that functions as a Drp1 receptor (40). The results of this study indicate that miR-200a-3p is bound to 3 'untranslated region ( $3^{\prime} U T R$ ) of MFF mRNA and decreased MFF expression. Ectopic expression of miR-200a-3p in Hep3B cells enhanced mitochondria elongation and increased mitochondrial activity without changes of other regulatory proteins including DRP1, MFN1/2, and OPA1. Our results suggest that miR-200a-3p functions as a novel factor regulating mitochondrial dynamics by decreasing MFF expression. 


\section{RESULTS}

miR-200a-3p is a novel factor regulating MFF expression Mitochondria dynamics is tightly regulated by several key proteins including DRP1, OPA1, MFN1/2 and MFF $(3,6)$. It has been reported that expression and activity of those key regulators are modulated via multiple steps including transcriptional, translational, post-transcriptional, and post-transitional modification. Previous studies have reported that miR-27, miR-593-5p, and miR-761 regulate MFF expression (38, 39, 41). In this study, we identified miR-200a-3p as a novel regulator governing MFF expression. A survey using two different miRNA prediction algorithms, Targetscan and microrna.org, revealed that MFF mRNA $3^{\prime} U T R$ has a potential binding site for miR-200a-3p (Fig. 1A). To investigate whether miR-200a-3p affects MFF expression, MFF mRNA and proteins levels were determined by RT-qPCR and Western blotting after miR200a-3p transfection. As shown in Fig. 1B, MFF mRNA level did not change by miR-200-3p. However, miR-200a-3p

A

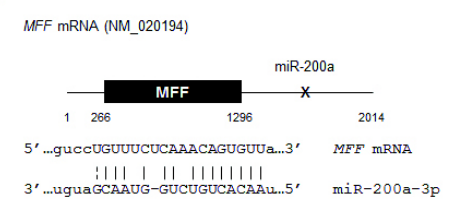

B

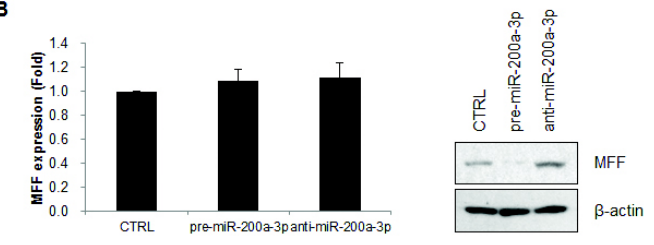

C

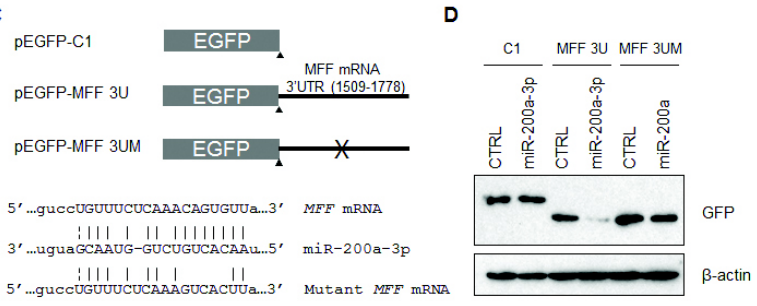

Fig. 1. miR-200a-3p down-regulated MFF expression. (A) Schematic diagram of MFF mRNA having miR-200a-3p binding site. (B) Hep3B cells were transfected with pre-miR-200a-3p, anti-miR-200a$3 p$, and control miRNA (CTRL). Forty-eight hours after transfection, abundance of MFF mRNA and protein were analyzed by RTqPCR and western blotting, respectively. (C) Schematic diagrams of the reporter plasmids pEGFP-C1 (control), pEGFP-MFF 3U, and pEGFP-MFF 3UM that lack miR-200a-3p binding site in in the MFF mRNA. (D) After transfection of miRNAs and EGFP reporters, GFP expression levels were analyzed by western blotting. Results are representative of three independent experiments. overexpression decreased MFF protein, and inhibition of miR-200a-3p increased it. To further analyze the regulation of MFF expression by miR-200a-3p, EGFP reporter was constructed by inserting MFF 3'UTR (1509-1778 nt) at the 3'UTR of EGFP open reading frame and EGFP levels were assessed after miR-200a-3p expression. miR-200a-3p downregulated the reporter expression containing MFF $3^{\prime} U T R$, but not that of mutant reporter that missing the seed region for miRNA binding (Fig. 1C and D). These results suggest that miR-200a-3p is responsible for MFF downregulation.

\section{miR-200a-3p increases mitochondrial elongation by MFF downregulation}

To investigate the effect of miR-200a-3p on the morphological changes of in mitochondria, we observed mitochondria morphology of CHANG cells expressing mtYFP or Hep3B cells incubated with Mitotracker, after regulation of miR-200a-3p level. As shown in Fig. $2 A$ and B, ectopic expression of miR-200a-3p increased the number of cells having elongated mitochondria, whereas miR-200a-3p inhibition increased the
A

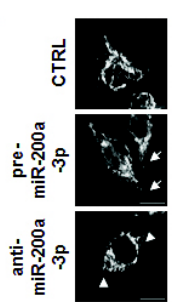

C

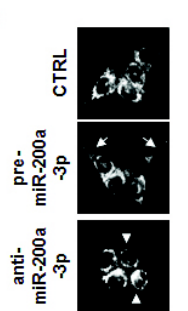

B

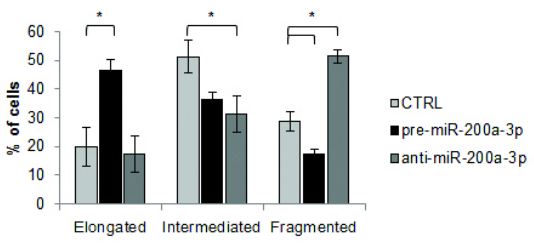

D

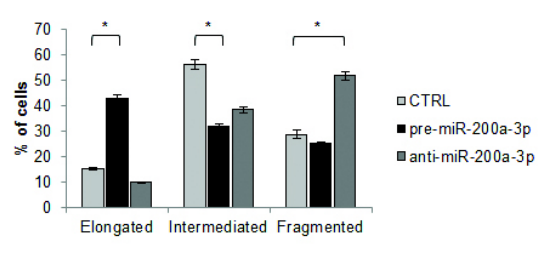

Fig. 2. miR-200a-3p inhibited mitochondria fission. (A) CHANGmtYFP cells were transfected with pre-miR-200a-3p, anti-miR-200a-3p, and control miRNA (CTRL). Forty-eight hours after transfection, mitochondrial morphology was observed by tracing YFP signals. (B) The number of cells was counted and grouped into three different categories according to mitochondrial morphology (intermediate, elongated or fragmented forms) from 100 cells. (C) Hep3B cells were transfected with pre-miR-200a-3p, anti-miR-200a-3p, and control miRNA (CTRL). After transfection of miRNAs, mitochondria were stained with MitoTracker and mitochondrial morphology was observed using a fluorescence microscope. (D) The number of cells were analyzed as described in (B). Images are representative of three independent experiments and the data represent the mean \pm SEM from three independent experiments. Arrows indicate elongated form of mitochondria and arrow heads indicated fragmented mitochondria. $* P<0.05$. 
portion of cells having fragmented mitochondria in CHANG mtYFP cells. The regulation of mitochondria morphology by miR-200a-3p was further analyzed in Hep3B cells. As shown in Fig. 2C and D, miR-200a-3p also increased the number of cells having elongated mitochondria of Hep3B cells.

Next, the effect of miR-200a-3p affected the levels of key proteins governing mitochondrial dynamics was investigated. The levels of DRP1, MFN1/2, and OPA1 did not change after upregulation or inhibition of miR-200a-3p (Fig. 3). Taken together, those results indicate that miR-200a-3p promotes mitochondrial elongation via MFF downregulation.

\section{miR-200a-3p enhances mitochondrial activity}

Morphological changes of mitochondria directly affect mitochondrial function including cellular respiration, ATP synthesis, reactive oxygen species production, and mitochondrial-mediated apoptosis (42-45). We investigated whether miR-200a-3p changes the mitochondrial activity. Mitochondrial ATP synthesis and membrane potential were assessed by Toxglo assay and JC1 staining after ectopic expression of miR-200a-3p. As shown in Fig. 4A, miR-200a-3p overexpression increased mitochondrial ATP synthesis and membrane potential. These results suggest that miR-200a-3p positively regulates the mitochondrial activity. In addition, oxygen consumption rate was also measured in Hep3B cells transfected with miR-200a-3p using a Seahorse FX analyzer. miR-200a-3p increased the basal respiration rate of mitochondria (Fig. 4B). These results indicate that miR-200a-3p has a potential to increase the mitochondrial activity via MFF downregulation.

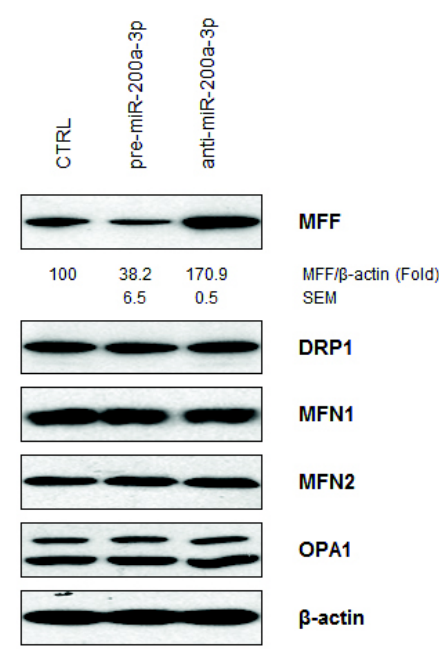

Fig. 3. Expression of DRP1, MFN1/2, and OPA1 were not changed by miR-200a-3p. CHANG-mtYFP cells were transfected with premiR-200a-3p, anti-miR-200a-3p and control miRNA (CTRL). Fortyeight hours after transfection, MFF, DRP1, MFN1/2, and OPA1 proteins were analyzed by western blotting. Results are representative of three independent experiments.

\section{DISCUSSION}

Fine-tuning of mitochondrial morphology is a critical step to maintain cellular homeostasis, and impaired regulation of mitochondrial dynamics leads to mitochondrial dysfunction that is responsible for the pathogenesis of several diseases such as cancer, neurodegenerative diseases, cardiovascular diseases $(7,46-48)$. Previous studies have shown that epigenetic and post-translational modifications are important regulatory mechanisms to control the quality of key proteins control mitochondrial dynamics (10-12, 31, 49). In addition, several studies have indicated that miRNAs are one of critical regulators governing the morphological changes of mitochondria $(29,31-36,38,41,50)$. In this study, we identified miR-200a-3p as a novel regulator of mitochondrial dynamics by targeting MFF.

miR-200a-3p is a member of miR-200 family consisting of miR-200a, miR-200b, miR-200c, miR-141, and miR-429. miR-200 family play a role in the regulation of cancer progression by targeting zinc finger E-box-binding homeobox $1 / 2$ (ZEB1/2) (51-55). miR-200a-3p is differentially expressed in various types of cancers and functions as a potential therapeutic target $(56,57)$. Besides tumor suppressive roles of miR-200a-3p, functional studies of miR-200a-3p are not fully elucidated. Herein, we found that miR-200a-3p is involved in the mitochondrial quality control by enhancing mitochondrial elongation. Ectopic expression of miR-200a-3p downregulated

A
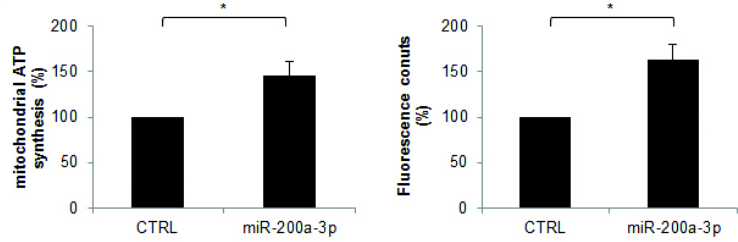

B

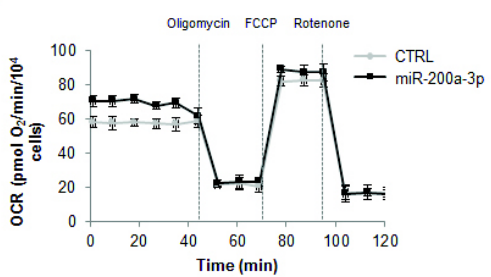

Fig. 4. miR-200a-3p affected mitochondrial function by regulating MFF expression. (A) Hep3B cells were transfected with miR200a-3p or control miRNA, and stained with ATP detection reagent (left) and JC-1 dye (right) to determine mitochondrial membrane potential and mitochondrial ATP levels. Change in the relative luminescence was assessed by measuring the fluorescence. Data represent the mean \pm SEM from three independent experiments. (B) Oxygen consumption rates (OCR) in miRNA transfected cells were analyzed using a Seahorse XF analyzer. Data represent the mean \pm SEM from three independent measurements. $* P<0.05$. 
MFF level (Fig. 1) and promoted mitochondrial elongation thereby increasing mitochondrial membrane potential and basal respiratory rate (Fig. 2 and 4) Although several reports have shown differential expression of miR-200a-3p in some types of disease models (58-62), the correlation between miR-200a-3p and mitochondrial dynamics in those models has not yet investigated in this study. Further studies are needed to confirm the implication of miR-200a-3p/MFF axis in the pathogenesis of human diseases.

\section{MATERIALS AND METHODS}

\section{Cell culture, transfection, plasmids and miRNAs}

Human CHANG liver cells that stably express yellow fluorescent protein, targeting mitochondria (CHANG-mtYFP cells) and Hep3B cells were cultured in Dulbecco's modified Eagle's medium (Invitrogen) contained with 10\% fetal bovine serum and $1 \%$ antibiotics. For reporter analysis, Enhanced green fluorescent protein (EGFP) reporter vectors were constructed by inserting 3'UTR region of MFF mRNA (1509-1778 bp) into pEGFP-C1 (BD Bioscience) (41). A mutant reporter lacking the binding sites for the miR-200a-3p seed region was generated by site-directed mutagenesis using a KOD-PlusMutagenesis Kit (Toyobo). miRNAs (Bioneer) were transiently transfected using Lipofectamine 2000 (Invitrogen).

\section{Western blot analysis}

Cells were lysed in RIPA buffer $(10 \mathrm{mM}$ Tris- $\mathrm{HCl}(\mathrm{pH} 7.4)$, $150 \mathrm{mM} \mathrm{NaCl}, 1 \% \mathrm{NP}-40,1 \mathrm{mM}$ EDTA and $0.1 \%$ sodium dodecyl sulfate) and analyzed by SDS-PAGE. Transferred membranes were incubated with primary antibodies against MFF (Abcam), GFP (Santa Cruz Biotech), MFN1 (Abcam), MFN2 (Sigma Aldrich), OPA1 (BD Bioscience), or $\beta$-actin (Abcam), and further incubated with appropriate secondary antibodies conjugated to horseradish peroxidase (Santa Cruz Biotech). Chemiluminescent signals were developed using Clarity ${ }^{\text {TM }}$ Western ECL substrate (Bio-Rad).

\section{Fluorescence microscopy}

Mitochondrial morphologies were observed under a fluorescence microscope, Axiovert 200M microscope (Carl Zeiss). Yellow fluorescence from mtYFP or red fluorescence from MitoTracker Red CMXRos (Invitrogen) was analyzed as described by Tak et al. (41). Images were acquired using an Axiovertcam mRM camera attached to Axiovert 200M microscope (Carl Zeiss). Mitochondrial length was determined by analyzing random 100 cells images of the cells transfected with mtYFP or stained with MitoTracker using Image J software.

\section{Measurement of the mitochondrial membrane potential and ATP level}

Mitochondrial membrane potential or mitochondrial ATP levels were determined using a JC1 Mitochondrial Membrane
Potential Assay Kit (Abcam) or the Mitochondrial ToxGlo assay (Promega) according to the manufacturer's protocol (41).

\section{Analysis of oxygen consumption}

Oxygen consumption rate (OCR) was assessed by Seahorse FX24 Extracellular Flux Analyzer (Seahorse Bioscience) according to the manufacturer's instruction. The number of cells $\left(1 \times 10^{3}\right)$ was used for OCR measurement. Basal OCR was measured for $3 \mathrm{~min}$ every $8 \mathrm{~min}$ for four points. Small molecule-metabolic modulators oligomycin $(3 \mu \mathrm{M})$, FCCP $(1$ $\mu \mathrm{M})$, and antimycin $\mathrm{A}(1 \mu \mathrm{M})$ were injected sequentially at the indicated time points after baseline OCR measurement.

\section{ACKNOWLEDGEMENTS}

This work is supported by the National Research Foundation of Korea (NRF) grant funded by the Korea government (2014 R1A2A1A11053431, 2012M3A9D1054517, and 2012R1A5A 2047939).

\section{CONFLICTS OF INTEREST}

The authors have no conflicting financial interests.

\section{REFERENCES}

1. Detmer SA and Chan DC (2007) Functions and dysfunctions of mitochondrial dynamics. Nat Rev Mol Cell Bio 8, 870-879

2. Suen D-F, Norris KL and Youle RJ (2008) Mitochondrial dynamics and apoptosis. Genes Dev 22, 1577-1590

3. Bereiter-Hahn J and Jendrach M (2010) Mitochondrial dynamics. Int Rev Cell Mol Biol 284, 1-65

4. Ni HM, Williams JA and Ding WX (2015) Mitochondrial dynamics and mitochondrial quality control. Redox Biol 4, 6-13

5. Zorzano A, Liesa M, Sebastian D, Segales J and Palacin M (2010) Mitochondrial fusion proteins: dual regulators of morphology and metabolism. Semin Cell Dev Biol 21, 566-574

6. Hyde BB, Twig G and Shirihai OS (2010) Organellar vs cellular control of mitochondrial dynamics. Semin Cell Dev Biol 21, 575-581

7. Kuzmicic J, Del Campo A, Lopez-Crisosto C et al (2011) [Mitochondrial dynamics: a potential new therapeutic target for heart failure]. Rev Esp Cardiol 64, 916-923

8. Gomes LC and Scorrano L (2013) Mitochondrial morphology in mitophagy and macroautophagy. Biochim Biophys Acta 1833, 205-212

9. Chang CR and Blackstone C (2010) Dynamic regulation of mitochondrial fission through modification of the dynaminrelated protein Drp1. Ann NY Acad Sci 1201, 34-39

10. Kashatus JA, Nascimento A, Myers LJ et al (2015) Erk2 phosphorylation of Drp1 promotes mitochondrial fission and MAPK-driven tumor growth. Mol Cell 57, 537-551

11. Samant SA, Zhang HJ, Hong Z et al (2014) SIRT3 deacetylates and activates OPA1 to regulate mitochondrial 
dynamics during stress. Mol Cell Biol 34, 807-819

12. Escobar-Henriques M (2014) Mitofusins: ubiquitylation promotes fusion. Cell Res 24, 387-388

13. Ke XS, Liu CM, Liu DP and Liang CC (2003) MicroRNAs: key participants in gene regulatory networks. Curr Opin Chem Biol 7, 516-523

14. Ma J, Lin Y, Zhan M, Mann DL, Stass SA and Jiang F (2015) Differential miRNA expressions in peripheral blood mononuclear cells for diagnosis of lung cancer. Lab Invest 95, 1197-1206

15. Sun J, Sonstegard TS, Li C et al (2015) Altered microRNA expression in bovine skeletal muscle with age. Anim Genet 46, 227-238

16. Kaviani M, Azarpira N, Karimi $\mathrm{MH}$ and Al-Abdullah I (2016) The role of microRNAs in islet beta-cell development. Cell Biol Int 40, 1248-1255

17. Achkar NP, Cambiagno DA and Manavella PA (2016) miRNA Biogenesis: A Dynamic Pathway. Trends Plant Sci 21, 1034-1044

18. Shah MY, Ferrajoli A, Sood AK, Lopez-Berestein G and Calin GA (2016) microRNA Therapeutics in Cancer - An Emerging Concept. EBioMedicine 12, 34-42

19. Feng J, Xing W and Xie L (2016) Regulatory Roles of MicroRNAs in Diabetes. Int J Mol Sci 17

20. Catalanotto C, Cogoni C and Zardo G (2016) MicroRNA in Control of Gene Expression: An Overview of Nuclear Functions. Int J Mol Sci 17, 1712

21. Geiger J and Dalgaard LT (2017) Interplay of mitochondrial metabolism and microRNAs. Cell Mol Life Sci 74, 631-646

22. Moss EG (2002) MicroRNAs: hidden in the genome. Curr Biol 12, R138-140

23. Donzelli $S$, Cioce $M$, Muti $P$, Strano $S$, Yarden $Y$ and Blandino G (2016) MicroRNAs: Non-coding fine tuners of receptor tyrosine kinase signalling in cancer. Semin Cell Dev Biol 50, 133-142

24. Mishra P and Chan DC (2016) Metabolic regulation of mitochondrial dynamics. J Cell Biol 212, 379-387

25. Kamiya Y, Kawada J, Kawano $Y$ et al (2015) Serum microRNAs as Potential Biomarkers of Juvenile Idiopathic Arthritis. Clin Rheumatol 34, 1705-1712

26. Song MA, Paradis AN, Gay MS, Shin J and Zhang L (2015) Differential expression of microRNAs in ischemic heart disease. Drug Discov Today 20, 223-235

27. Irwandi RA and Vacharaksa A (2016) The role of microRNA in periodontal tissue: A review of the literature. Arch Oral Biol 72, 66-74

28. Ojha CR, Rodriguez M, Dever SM, Mukhopadhyay R and El-Hage N (2016) Mammalian microRNA: an important modulator of host-pathogen interactions in human viral infections. J Biomed Sci 23, 74

29. Fan S, Chen WX, Lv XB et al (2015) miR-483-5p determines mitochondrial fission and cisplatin sensitivity in tongue squamous cell carcinoma by targeting FIS1. Cancer Lett 362, 183-191

30. Wang K, Long B, Jiao JQ et al (2012) miR-484 regulates mitochondrial network through targeting Fis1. Nat Commun 3, 781

31. Wang JX, Jiao JQ, Li Q et al (2011) miR-499 regulates mitochondrial dynamics by targeting calcineurin and dynamin-related protein-1. Nat Med 17, 71-78

32. Guan X, Wang L, Liu Z et al (2016) miR-106a promotes cardiac hypertrophy by targeting mitofusin 2. J Mol Cell Cardiol 99, 207-217

33. Joshi SR, Dhagia V, Gairhe S, Edwards JG, McMurtry IF and Gupte SA (2016) MicroRNA-140 is elevated and mitofusin-1 is downregulated in the right ventricle of the Sugen5416/hypoxia/normoxia model of pulmonary arterial hypertension. Am J Physiol Heart Circ Physiol 311, H689-698

34. Zhang R, Zhou H, Jiang L et al (2016) MiR-195 dependent roles of mitofusin2 in the mitochondrial dysfunction of hippocampal neurons in SAMP8 mice. Brain Res 1652, 135-143

35. Zhou X, Zhang L, Zheng B et al (2016) MicroRNA-761 is upregulated in hepatocellular carcinoma and regulates tumorigenesis by targeting Mitofusin-2. Cancer Sci 107, 424-432

36. Li X, Wang FS, Wu ZY, Lin JL, Lan WB and Lin JH (2014) MicroRNA-19b targets Mfn1 to inhibit Mfn1-induced apoptosis in osteosarcoma cells. Neoplasma 61, 265-273

37. Zhou X, Zuo S and Xin W (2015) miR-27b overexpression improves mitochondrial function in a Sirt1-dependent manner. J Physiol Biochem 71, 753-762

38. Fan S, Liu B, Sun L et al (2015) Mitochondrial fission determines cisplatin sensitivity in tongue squamous cell carcinoma through the BRCA1-miR-593-5p-MFF axis. Oncotarget 6, 14885-14904

39. Long B, Wang K, Li N et al (2013) miR-761 regulates the mitochondrial network by targeting mitochondrial fission factor. Free Radic Biol Med 65, 371-379

40. Toyama EQ, Herzig S, Courchet J et al (2016) Metabolism. AMP-activated protein kinase mediates mitochondrial fission in response to energy stress. Science 351, 275-281

41. Tak H, Kim J, Jayabalan AK et al (2014) miR-27 regulates mitochondrial networks by directly targeting the mitochondrial fission factor. Exp Mol Med 46, e123

42. Bose A and Beal MF (2016) Mitochondrial dysfunction in Parkinson's disease. J Neurochem 139 Suppl 1, 216-231

43. Lee $\mathrm{H}$ and Yoon $\mathrm{Y}$ (2016) Mitochondrial fission and fusion. Biochem Soc Trans 44, 1725-1735

44. Silva Ramos E, Larsson NG and Mourier A (2016) Bioenergetic roles of mitochondrial fusion. Biochim Biophys Acta 1857, 1277-1283

45. Wada J and Nakatsuka A (2016) Mitochondrial Dynamics and Mitochondrial Dysfunction in Diabetes. Acta Med Okayama 70, 151-158

46. Chen H and Chan DC (2009) Mitochondrial dynamicsfusion, fission, movement, and mitophagy-in neurodegenerative diseases. Hum Mol Genet 18, R169-R176

47. Cho DH, Nakamura $T$ and Lipton SA (2010) Mitochondrial dynamics in cell death and neurodegeneration. Cell Mol Life Sci 67, 3435-3447

48. Rehman J, Zhang HJ, Toth PT et al (2012) Inhibition of mitochondrial fission prevents cell cycle progression in lung cancer. FASEB J 26, 2175-2186

49. Saita S, Ishihara T, Maeda M et al (2016) Distinct types of protease systems are involved in homeostasis regulation of mitochondrial morphology via balanced fusion and fission. Genes Cells 21, 408-424 
50. Xu Y, Zhao C, Sun X, Liu Z and Zhang J (2015) MicroRNA-761 regulates mitochondrial biogenesis in mouse skeletal muscle in response to exercise. Biochem Biophys Res Commun 467, 103-108

51. Brabletz S and Brabletz T (2010) The ZEB/miR-200 feedback loop-a motor of cellular plasticity in development and cancer? EMBO Rep 11, 670-677

52. Bracken CP, Gregory PA, Kolesnikoff $N$ et al (2008) A double-negative feedback loop between ZEB1-SIP1 and the microRNA-200 family regulates epithelial-mesenchymal transition. Cancer Res 68, 7846-7854

53. Hill L, Browne G and Tulchinsky E (2013) ZEB/miR-200 feedback loop: at the crossroads of signal transduction in cancer. Int J Cancer 132, 745-754

54. Cong N, Du P, Zhang A et al (2013) Downregulated microRNA-200a promotes EMT and tumor growth through the wnt/beta-catenin pathway by targeting the E-cadherin repressors ZEB1/ZEB2 in gastric adenocarcinoma. Oncol Rep 29, 1579-1587

55. Sulaiman SA, Ab Mutalib NS and Jamal R (2016) miR-200c Regulation of Metastases in Ovarian Cancer: Potential Role in Epithelial and Mesenchymal Transition. Front Pharmacol 7, 271

56. Diaz T, Tejero R, Moreno I et al (2014) Role of miR-200 family members in survival of colorectal cancer patients treated with fluoropyrimidines. J Surg Oncol 109, 676683
57. Koutsaki M, Spandidos DA and Zaravinos A (2014) Epithelial-mesenchymal transition-associated miRNAs in ovarian carcinoma, with highlight on the miR-200 family: prognostic value and prospective role in ovarian cancer therapeutics. Cancer Lett 351, 173-181

58. Chen C, Yang D, Wang Q and Wang X (2015) Expression and Clinical Pathological Significance of miR-200a in Concurrent Cholangiocarcinoma Associated with Hepatolithiasis. Med Sci Monit 21, 3585-3590

59. Dhayat SA, Mardin WA, Kohler G et al (2014) The microRNA-200 family-a potential diagnostic marker in hepatocellular carcinoma? J Surg Oncol 110, 430-438

60. Leskela S, Leandro-Garcia LJ, Mendiola M et al (2011) The miR-200 family controls beta-tubulin III expression and is associated with paclitaxel-based treatment response and progression-free survival in ovarian cancer patients. Endocr Relat Cancer 18, 85-95

61. Li H, Tang J, Lei H et al (2014) Decreased MiR-200a/141 suppress cell migration and proliferation by targeting PTEN in Hirschsprung's disease. Cell Physiol Biochem 34, 543-553

62. Zuberi M, Mir R, Das J et al (2015) Expression of serum miR-200a, miR-200b, and miR-200c as candidate biomarkers in epithelial ovarian cancer and their association with clinicopathological features. Clin Transl Oncol 17, 779-787 